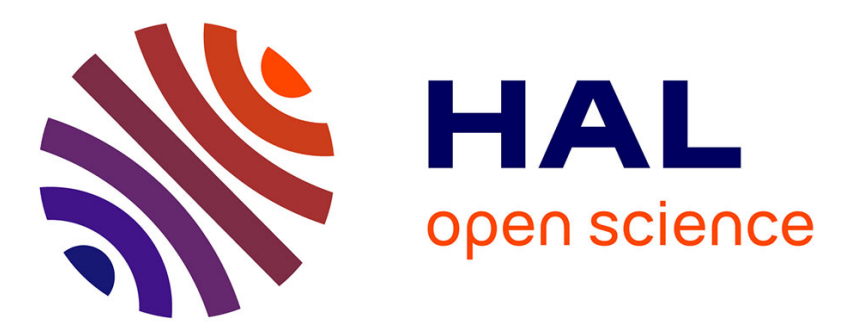

\title{
The conceptualization of a Just-In-Time Adaptive Intervention (JITAI) for the reduction of sedentary behavior in older adults
}

\author{
Andre Matthias Müller, Ann Blandford, Lucy Yardley
}

\section{- To cite this version:}

Andre Matthias Müller, Ann Blandford, Lucy Yardley. The conceptualization of a Just-In-Time Adaptive Intervention (JITAI) for the reduction of sedentary behavior in older adults. mHealth, 2017, 3, pp.37. 10.21037/mhealth.2017.08.05 . hal-01598199

\author{
HAL Id: hal-01598199 \\ https://hal.science/hal-01598199
}

Submitted on 29 Sep 2017

HAL is a multi-disciplinary open access archive for the deposit and dissemination of scientific research documents, whether they are published or not. The documents may come from teaching and research institutions in France or abroad, or from public or private research centers.
L'archive ouverte pluridisciplinaire HAL, est destinée au dépôt et à la diffusion de documents scientifiques de niveau recherche, publiés ou non, émanant des établissements d'enseignement et de recherche français ou étrangers, des laboratoires publics ou privés. 


\title{
The conceptualization of a Just-In-Time Adaptive Intervention (JITAI) for the reduction of sedentary behavior in older adults
}

\author{
Andre Matthias Müller ${ }^{1}$, Ann Blandford ${ }^{2}$, Lucy Yardley ${ }^{1,3}$ \\ ${ }^{1}$ Centre for Clinical and Community Applications of Health Psychology (CCCAHP), School of Psychology, Faculty of Social, Human and \\ Mathematical Sciences, University of Southampton, Southampton, UK; ${ }^{2}$ Institute of Digital Health, University College London, London, UK; \\ ${ }^{3}$ Nuffield Department of Primary Care Health Sciences, University of Oxford, Oxford, UK \\ Contributions: (I) Conception and design: All authors; (II) Administrative support: All authors; (III) Provision of study material or patients: All authors; \\ (IV) Collection and assembly of data: All authors; (V) Data analysis and interpretation: AM Müller, L Yardley; (VI) Manuscript writing: All authors; \\ (VII) Final approval of manuscript: All authors. \\ Correspondence to: Andre Matthias Müller. Centre for Clinical and Community Applications of Health Psychology (CCCAHP), School of Psychology, \\ Faculty of Social, Human and Mathematical Sciences, University of Southampton, Southampton, UK. Email: andrematthiasmueller@gmail.com.
}

\begin{abstract}
Low physical activity and high sedentary behavior in older adults can be addressed with interventions that are delivered through modern technology. Just-In-Time Adaptive Interventions (JITAIs) are an emerging technology-driven behavior-change intervention type and capitalize on data that is collected via mobile sensing technology (e.g., smartphones) to trigger appropriate support in real-life. In this paper we integrated behavior change and aging theory and research as well as knowledge around older adult's technology use to conceptualize a JITAI targeting the reduction of sedentary behavior in older adults. The JITAIs ultimate goal is to encourage older adults to take regular activity breaks from prolonged sitting. As a proximal outcome, we suggest the number of daily activity breaks from sitting. Support provided to interrupt sitting time can be based on tailoring variables: (I) the current accumulated sitting time; (II) the location of the individual; (III) the time of the day; (IV) the frequency of daily support prompts; and (V) the response to previous support prompts. Data on these variables can be collected using sensors that are commonly inbuilt into smartphones (e.g., accelerometer, GPS). Support prompts might be best delivered via traditional text messages as older adults are usually familiar and comfortable with this function. The content of the prompts should encourage breaks from prolonged sitting by highlighting immediate benefits of sitting time interruptions. Additionally, light physical activities that could be done during the breaks should also be presented (e.g., walking into the kitchen to prepare a cup of tea). Although the conceptualized JITAI can be developed and implemented to test its efficacy, more work is required to identify ways to collect, aggregate, organize and immediately use dense data on the proposed and other potentially important tailoring variables. Machine learning and other computational modelling techniques commonly used by computer scientists and engineers appear promising. With this, to develop powerful JITAIs and to actualize the full potential of modern sensing technologies transdisciplinary approaches are required
\end{abstract}

Keywords: Physical activity; eHealth; mHealth; behavior change; smartphone

Received: 09 June 2017; Accepted: 14 August 2017; Published: 12 September 2017.

doi: 10.21037/mhealth.2017.08.05

View this article at: http://dx.doi.org/10.21037/mhealth.2017.08.05

\section{Introduction}

Population aging is a phenomenon that can be observed in most regions of the world (1). According to the United Nations the proportion of older adults in the overall population will almost double from $12 \%$ in 2013 to $21 \%$ in 2050 (2). As older adults are more likely to develop chronic diseases such as diabetes, health care systems face an enormous challenge $(3,4)$. However, many chronic diseases 
can be prevented and good health can be maintained if older adults adopt healthy lifestyles. This commonly includes engaging in regular physical activity and reducing sedentary behavior $(5,6)$. For example, physical activity can lower the risk of cardiovascular diseases (7), improves memory and other cognitive functions (8-10), and increases wellbeing (11).

Unfortunately, older adults have low physical activity levels, which decline with increasing age (12). For example, recent accelerometer data from the United Kingdom suggests that only $13.5 \%$ of older adults meet the World Health Organizations recommendations for physical activity (150 minutes of moderate to vigorous physical activity per week) (13). In addition, older adults are the most sedentary age group who spend between $65 \%$ to $80 \%$ of their waking hours in sitting or reclining positions $(14,15)$.

To address low physical activity and high sedentary behavior levels in older adults, an increasing number of researchers employ electronic and mobile health (e\& mHealth) approaches to deliver behavior change interventions (16-18). This is reasonable considering that older adults are increasingly keen to adopt modern technologies (19-21). For example, about $80 \%$ of older adults are regular mobile phone users (22). Research also shows that older adults are especially interested in modern technologies if these can benefit important aspects of life, such as health $(23,24)$. As further increases in uptake and use of (mobile) technologies by older adults are expected (25), new avenues to deliver interventions targeting physical activity and sedentary behavior are evolving rapidly.

In this paper, we will first provide a brief overview of existing e- \& mHealth interventions that target physical activity and/or sedentary behavior in older adults before introducing a new intervention type, Just-In-Time Adaptive Interventions (JITAIs), which can enable sustainable health behavior change (26). The primary aim of this paper is to utilize theoretical and empirical evidence to conceptualize a JITAI that targets reduced sedentary behavior in older adults.

\section{E- \& mHealth to impact physical activity and sedentary behavior}

The evidence base for e- \& mHealth physical activity and sedentary behavior interventions in older adults is mixed. Some interventions delivered via mobile-phone text messaging $(27,28)$, and the Internet (29-33) were successful in improving these health behaviors. In contrast, others, delivered via the Internet $(34,35)$, tablet computers (36) and wearable activity trackers $(37,38)$, were not. Even if positive changes were reported, these were not always maintained for a long period following the intervention (39). Hence, although it appears feasible to use modern technology to deliver physical activity and sedentary behavior interventions targeting older adults, the inconclusive evidence highlights that the full potential of e- \& mHealth is yet to be actualized.

One way to create more successful interventions for reducing sedentary behavior and increase activity is to use sensors that are inbuilt into mobile devices such as smartphones (e.g., GPS, accelerometers). These sensors can capture behavioral and contextual data in ongoing interventions. This data can be aggregated and processed to inform and adapt behavior change support in real time $(40,41)$. With this, the type, time, frequency and content of support is not only dependent on baseline characteristics (e.g., age, sex) and theory. In fact, temporal dynamics of various factors that are likely to influence behavior "in the wild" (e.g., location, time of the day) inform the choice of behavior change support (42). As a result, appropriate support can be provided when it is most beneficial. This will likely lead to improved adoption and maintenance of the health behavior in question (26). Interventions that utilize continuously collected data to adapt behavior change support in the moment are called JITAIs $(43,44)$.

\section{JITAls to impact physical activity and sedentary behavior in older adults: current state}

There are only three JITAI studies that targeted physical activity and/or sedentary behavior which also included older adults (45-47). However, the researchers only used data from smartphone accelerometers to inform behavior change support. This is unsatisfying considering that (I) various contextual factors (e.g., the current location) can have a profound impact on behavior in the moment, and (II) data on these contextual factors can be collected and processed to identify moments where a certain type of behavioral support might be most beneficial $(43,48)$.

To further advance the field, and to develop strong JITAIs, it is important to integrate theoretical and empirical evidence and, in the process, to answer four essential questions:

(I) What behavioral and contextual data are needed to identify moments when the delivery of behavior change support will be most beneficial?

(II) What level of the behavioral and contextual data 


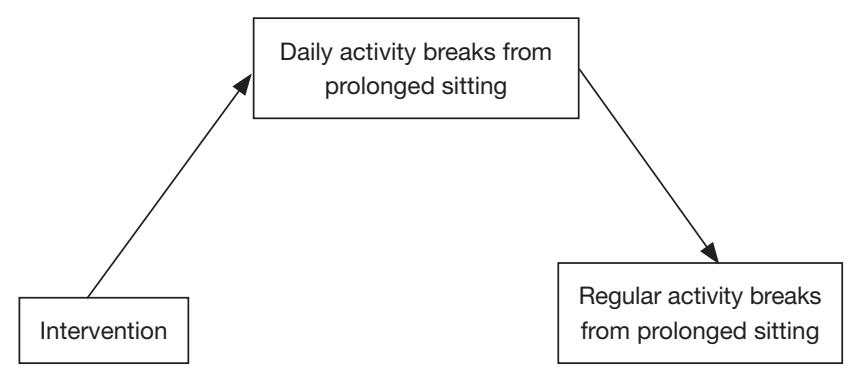

Figure 1 Intervention impact on distal outcome (regular activity breaks from prolonged sitting) via the proximal outcome (daily activity breaks from prolonged sitting)

indicates an opportunity to deliver behavior change support?

(III) What is the best way to measure the identified behavioral and contextual data?

(IV) What kind of support could be delivered in what way when opportune moments for behavior change support arise?

In this paper, we describe the integration of behavior change and aging theory as well as empirical data to inform the conceptualization of a JITAI for the reduction of sedentary behavior in older adults. We will also discuss older adults' adoption and use of technologies that are relevant for the JITAI.

\section{Theoretical and empirical evidence-informed conceptualization of a JITAI for sedentary behavior reduction in older adults}

A JITAI consists of six components: distal outcome, proximal outcome, tailoring variables, intervention options, decision points, and decision rules (26).

\section{Distal outcome}

The distal outcome is the long-term goal of a JITAI (44). For the purpose of this paper, the distal outcome is regular interruptions of prolonged sitting with light physical activity. We chose this outcome for four reasons.

First, although older adults have the lowest physical activity levels compared to other age groups and increasing moderate or vigorous activity is desirable $(12,49)$, meaningful improvements in physical activity might not be feasible because older adults face various age-related limitations $(10,50)$. Second, older adults are likely to be highly committed to less challenging behavior changes such as reducing sedentary behavior as these are in line with their current capabilities and competencies $(51,52)$. Third, there is evidence highlighting that sedentary behavior is associated with many health risks $(53,54)$, and that reducing sedentary behavior is beneficial for health $(55,56)$. Finally, sitting is habitual and active breaks are opportunistic and can be promoted in the moment (with a JITAI) $(57,58)$.

\section{Proximal outcome}

The proximal outcome is the short-term goal of a JITAI as it mediates the effect of the intervention on the distal outcome. Hence, the proximal outcome is at the center of a JITAI because it indicates progress towards the distal outcome (44).

There is only limited research on the mediators of sedentary behavior, especially in older adults $(45,57,59)$. Theory and evidence suggests that the social-cognitive determinants of self-efficacy and social support are important for physical activity adoption and maintenance (60-64). However, these determinants are not likely to influence how frequently an (older) adult takes active breaks from prolonged sitting because sedentary behaviors are habitual (65).

A behavioral proximal outcome that captures small steps towards sustainable behavior change (distal outcome) seems most appropriate (26). An adequate proximal outcome that is likely to mediate the effect on regular activity breaks from sitting is the number of active breaks from prolonged sitting over a day. Conceptually, when an older adult interrupts his/her sitting time throughout the day for a few weeks it is likely that breaks from sitting will soon become habitual. Figure 1 depicts the model.

\section{Tailoring variables}

To help an older adult to take active sitting breaks throughout the day it is important to collect data on tailoring variables that identify moments when behavioral support might be most beneficial (43). Sensors allow for the collection of behavioral and contextual data that can identify such moments. The choice of tailoring variables needs to be in line with theoretical and empirical evidence while also considering practical aspects (43).

The first tailoring variable is accumulated sitting time. This variable directly relates to the proximal outcome and was chosen in a JITAI that targeted sitting time reductions 


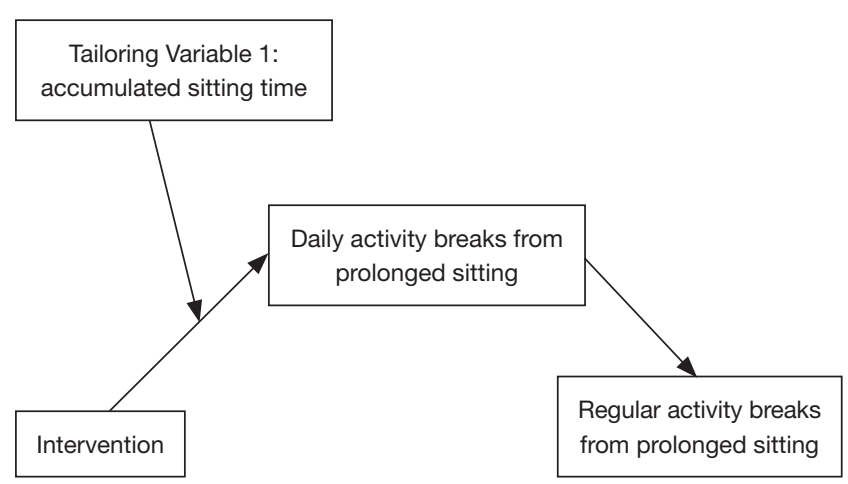

Figure 2 Relationship between the Tailoring Variable 1, the proximal outcome and the distal outcome

in office workers (66). The rationale behind choosing this tailoring variable is that after a longer time of sitting (e.g., 30 or 60 minutes) the individual might be receptive to the suggestion to take an active sitting break (67) (Figure 2).

The second tailoring variable is the location of the individual. Collecting data on this variable is important because sitting time can also accumulate outside the private home, at places where it is not possible to take active breaks. For example, sitting time interruptions in a bus or restaurant might not be possible or desirable. With this, promoting active breaks at home is likely most acceptable. In addition, research suggests that during the initial stages of forming a behavioral habit it is important to execute the behavior in a consistent setting (68). As older adults spend a considerable amount of time at home, providing support in this setting is likely to lead to faster habit formation.

A third tailoring variable that can guide the identification of opportune moments for the delivery of behavior change support is the time of day. There is evidence suggesting that older adults accumulate most of their sitting time in the evening (14,69-71). Older adults are also more inclined to take active sitting breaks in the evening hours compared to other parts of the day (72). Consequently, providing support in the evening is likely to be most effective.

The fourth tailoring variable is the response to support prompts sent earlier. If an older adult had an active sitting break following a support prompt it might not be desirable to send another one shortly afterwards. This is because the person might not be open to too many sitting time interruptions. It is important to consider that changes to habitual sedentary behaviors are difficult to achieve (51). It is likely that reducing sitting time in a step-by-step manner is more acceptable $(73,74)$. If the older adult does not interrupt his/her sitting time following a few support prompts it might not be desirable to continue providing prompts as these could be disturbing.

The final tailoring variable is frequency of support prompts. In order to minimize burden only a few support prompts per day should be delivered. Sending a maximum of three prompts per day proved successful in a previous JITAI (66).

Following the choice of adequate tailoring variables, it is essential to identify the technologies that could be used to collect data on these variables. To reduce participant burden, increase data accuracy, and allow for flexible data collection the tailoring variables should be measured automatically using sensors inbuilt into smartphones $(26,43)$. Although smartphone adoption among older adults is still lower compared to other age groups $(75,76)$ and older adults only use a few functions (19) this is projected to change over the coming years (25).

Smartphone inbuilt accelerometers or inclinometers can measure the tailoring variables accumulated sitting time and responses to previous support prompts. It might be beneficial to additionally use wearable activity trackers because older adults are unlikely to carry their smartphone around throughout the day, especially when they are at home. Research suggests that older adults accept such trackers well $(37,77)$ and that they find them easy to use (78). However, uptake of such devices is low (20) and it might be necessary to provide some training to familiarize older adults with this technology $(46,47)$. The location can be measured with the inbuilt GPS and the time of the day with the inbuilt clock.

\section{Intervention options}

Once the tailoring variables identify an opportune moment for behavior change support, adequate intervention options (support prompts) need to be triggered (44). The Socioemotional Selectivity Theory suggests that older adults value immediate and personally relevant benefits of behavior because the prominent perception is that their lifespan is limited $(79,80)$. This was confirmed in studies on the motivators for increasing physical activity and reducing sedentary behavior; the researchers reported that long-term health outcomes were less relevant compared to immediate benefits such as joy and well-being $(51,60)$. In addition to highlighting immediate benefits, it is important to provide activity options for the active breaks (e.g., walking to the kitchen to prepare a cup of tea) (51). Thus, Intervention Option 1 should be to provide a brief prompt that a) 


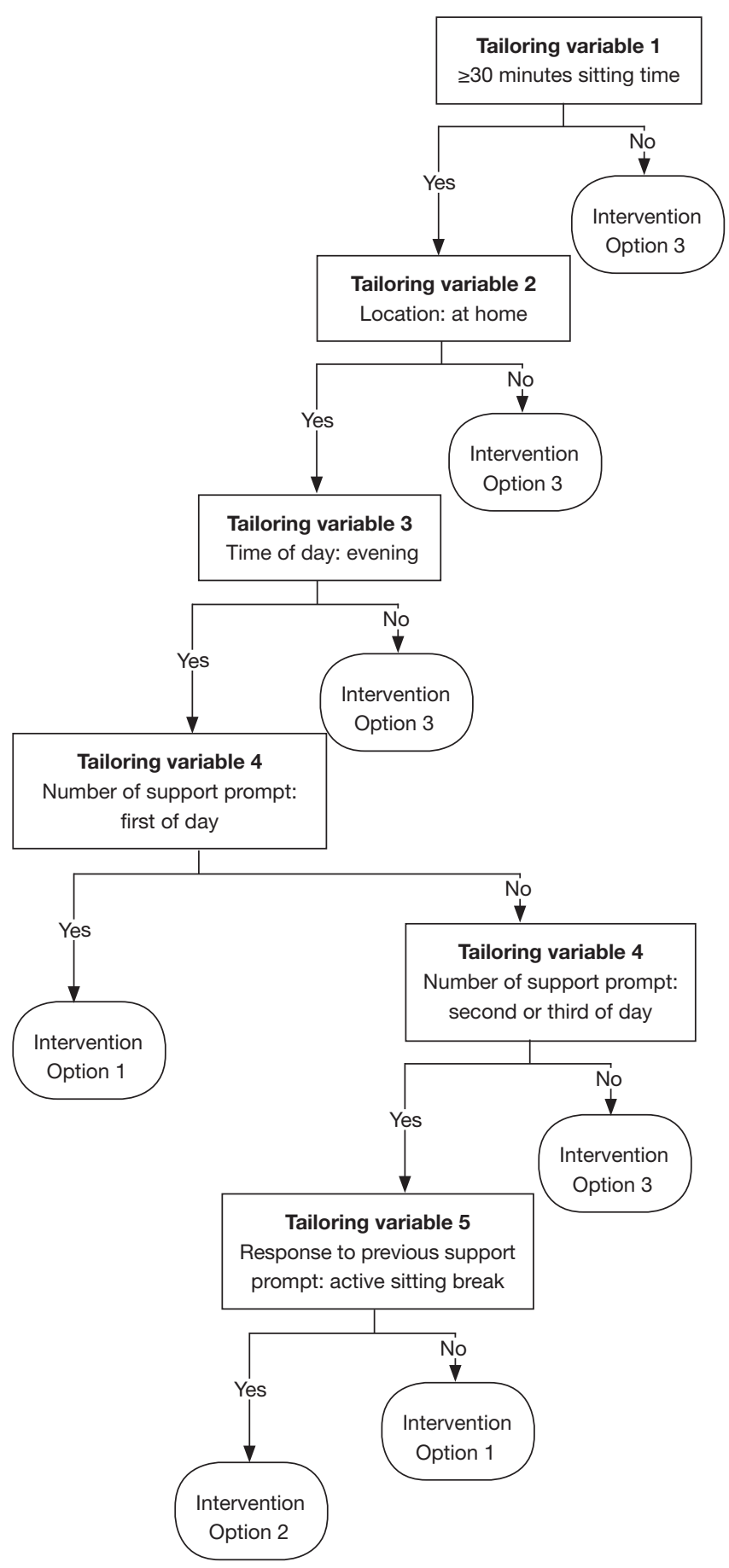

Figure 3 Theoretical and empirical evidence-informed conceptualization of a JITAI to reduce sitting time in older adults JITAI, Just-In-Time Adaptive Intervention. highlights the immediate benefits of taking an active sitting break, and b) proposes an activity for the break.

If sitting time was interrupted successfully following an earlier prompt, then the next prompt should provide positive feedback and encouragement to repeat the behavior (Intervention Option 2). The Provide Nothing Option (Intervention Option 3) is important in case sitting time cannot be interrupted in the moment (e.g., person is traveling on a bus) or when the individual might be unmotivated to interrupt his/her sitting time (e.g., after many sitting time interruptions earlier on the same day).

Intervention Options 1 and 2 could be delivered via simple text messages because older adults are usually familiar with this less complex technology (22). Using more recent technologies for delivery such as social media messengers might be problematic as most older adults are less comfortable with such technologies (19,76). An audible signal should alert the individual to the arrival of a text message.

\section{Decision points}

The decision points mark the moments in time when a decision about an intervention option is made based on the level of the tailoring variables $(26,44)$. Hence, decision points are intrinsically linked to the tailoring variables. As highlighted above, the evening hours are the time period when older adults accumulate most of their sitting time, and when they are most likely to be able and willing to take sitting breaks $(70,72)$. Consequently, the decisions points should be in the evening hours (potentially between $5 \mathrm{pm}$ to $9 \mathrm{pm}$ ). Tailoring variables 1, 2, 4 and 5 will then determine when an intervention is triggered. Figure 3 depicts the complete conceptual JITAI and a full description of the components can be found in the Table S1.

\section{Decision rules}

Decision rules systematically link the components of a JITAI $(26,44)$. Based on the five tailoring variables and the three intervention options we constructed seven decision rules. For example, if an older adult accumulates at least 30 minutes of uninterrupted sitting time but he/she is not at home, Intervention Option 3 should be triggered (provide nothing, Decision Rule 2). In contrast, if an older adult accumulates at least 30 minutes of uninterrupted sitting time and (I) he/she is at home, (II) it is evening, (III) the second support prompt of the day is due and (IV) the person did not interrupt his/her sitting time following the previous 
prompt then Intervention Option 1 should be delivered (support prompt, Decision Rule 6). See Figure 3 and Table S1 for a detailed overview of the decision rules.

\section{Discussion}

JITAIs are a novel intervention type that capitalize on sensing technology inbuilt into mobile devices (e.g., smartphones) to collect real-time behavioral and contextual data which is used to provide in-the-moment behavioral support $(26,43,44)$. These interventions have been successful in increasing physical activity and/or reducing sedentary behavior in a few small-scale studies involving adults $(66,81)$ and older adults $(45-47)$. The changes in sedentary behavior were moderate and mostly comparable to the changes achieved in other interventions that did not apply a JITAI approach or were not delivered digitally $(57,69,72,74,82)$. The shortcomings of the JITAI studies were that the integration of theory and empirical evidence to inform the development of the JITAIs was limited. As a result, the full potential of the JITAI approach was not actualized which might explain the modest effects.

In this paper we conceptualized a JITAI that targets reduced sitting time in older adults. For this, we integrated behavior change as well as aging theory and evidence, and also considered older adults' technology adoption and use. We hope our work can serve as a solid framework for the design of future JITAIs that could be effective in reducing sedentary behavior.

Although we carefully integrated theory and empirical research to conceptualize the JITAI it is important to highlight that not all tailoring variables that might indicate opportune moments for behavior change support could be considered. We focused on tailoring variables that can be assessed in a passive and unobtrusive manner using smartphones and their inbuilt sensors (e.g., GPS). This choice was made because passive measurement minimizes burden and allows for flexible as well as accurate assessments of the momentary context (43). We acknowledge that other tailoring variables than those included in our JITAI are likely to be also important. For example, the current internal state (happy, annoyed or energized) might be a promising tailoring variable to identify moments in which behavior support can be beneficial or, conversely, detrimental (43). If an older adult had some negative experiences he/she might have negative mood. In such situations providing behavior change support could be perceived negatively, even though all other tailoring variables point to an opportune moment for such support. However, assessing mood might not be desirable because the older adult would most likely be required to input some information into the mobile device. This would increase burden and might lead to disengagement with the intervention (44). Additionally, the complex and time-varying interactions between various behavioral and contextual tailoring variables which impact behavior were not considered in our work. This is mainly because these interactions and their implications are difficult to anticipate without dense data $(41,44)$.

In addition to adaptations based on the momentary context, JITAIs are meant to adapt to the progress towards the proximal and distal outcome (43). For example, if an older adult successfully interrupted his/her sitting time in the evening hours for a few weeks, the frequency of support prompts could be increased to encourage further reductions in sitting time. That means that the decision rules proposed here will need to change over time (43). When such adaptations to decisions rules should be made is not clear. There is limited evidence originating from one study that suggests that the adoption of a healthy habit can take between 18 and 254 days (median: 66 days) (68). This study provides little guidance on when decision rules should be adapted in a JITAI targeting sedentary behavior in older adults because (I) the researchers included younger adults who were motivated to change their behaviors, and (II) the reduction of sedentary behavior was not assessed. Older adults might need a longer time to adopt a new behavior due to long-established behavioral patterns. On the contrary, reducing sedentary time is not as complex as increasing physical activity. With this, habit formation might not take very long. The fact that the JITAI studies published to date described minimal $(46,47)$ or even no adaptations $(45,66,81)$ to decision rules highlights that not all opportunities that JITAIs offer are currently utilized.

As JITAIs capitalize on mobile technology, questions around feasibility and cost-effectiveness to implement largescale interventions in older adults need to be answered. Although the 'digital divide' is narrowing, older adults show lower technology adoption and use compared to other age groups $(22,78)$. This is especially true for recent technologies which bear most promise for JITAIs. This has implications for the design of JITAIs as it might be necessary to train older adults in using these technologies (24). In the three JITAI studies that included older adults, training sessions prior to the interventions were conducted to familiarize the participants with the technologies (45-47). For example, King and colleagues provided a 1-hour one- 
to-one training session for each participant $(46,47)$. A helpline for participants was also established. Even more support might be necessary for the older adults who experience more barriers to technology use (78). Providing such support on a larger scale might be impractical and cost-ineffective. On the contrary, if older adults successfully adopt modern mobile technologies, JITAIs might be a promising way to improve long-term impact on behavior. This is because older adults are less likely to abandon these technologies (78) and are more adherent to digital and other health interventions compared to younger age groups $(29,83)$. There is another benefit of a face-to-face introductory session prior to a JITAI: Older adults can be made aware of their sedentary behavior and intentions to reduce time spent sedentary can be formed (57). The JITAI can then function as a bridge between intentions and behavior. This will likely increase the sustainability of behavior change.

\section{Future work}

JITAI research is in its infancy (43) and the JITAI conceptualized in this paper presents a necessary step for the development and implementation of such interventions. The JITAI proposed here is limited as it does not account for (I) all potentially important factors that can impact behavior, (II) time-varying changes to these factors, and (III) complex and changing interactions between these factors and behavior.

Understanding these complexities and theorizing them solely from a behavioral science point of view is neither feasible nor fruitful as they vary from individual to individual. To refine the JITAI and to ensure effective behavior change, temporally dense longitudinal data of important variables is required that can be fed back into the JITAI to allow for ongoing adaptations at the individual level (feedback loop) (41). Such strategies of data aggregation, connection and organization for continuous model refinement are commonly applied in computer science and engineering and are often referred to as computational modelling (such as machine learning or system identification) $(40,41)$.

With advancing sensor technology that is increasingly integrated in smartphones and other consumer-based mobile devices, and the use of machine learning approaches, it is possible to understand and impact individual behavior in real-time (41). This is likely to significantly change behavioral health research and practice as it will allow for the generation of behavioral theories and models that are informed by classical theories (that are concerned with more stable factors), refined by data (of dynamic factors), and individually adaptable. Such work requires transdisciplinary teams involving behavioral health experts, engineers and computer specialists $(40,43,84)$. Finally, it is essential to consider the ethical implications related to the collection of dense personal data necessary for adaptive interventions.

\section{Conclusions}

Health behavior change intervention research and practice is maturing rapidly. From the early days where interventions were generic and intended to help all people in all contexts, to interventions that were tailored based on baseline data, we are now approaching the era of behavior change interventions, such as JITAIs, that are continuously adapted based on data collected through modern sensing technology (42).

The primary merit of JITAIs is that they recognize and make use of the momentary context that can have a profound impact on the adoption and maintenance of health behaviors. Modern technology enables the collection of information on the behavioral context; and this information can then be used to trigger immediate behavior change support. In this paper we integrated behavioral health and aging theory as well as knowledge around the use of technology by older adults to arrive at a conceptual JITAI targeting the replacement of sitting time with light physical activity. This JITAI could be further refined before piloting it. Advancements will be made if dense contextual data is collected and fed back into the JITAI (i.e., via machine learning). This will only be possible if transdisciplinary teams consisting of behavioral scientists, engineers and computer specialists work together.

Finally, despite the great opportunities of JITAIs participants need to be inclined to and experienced in using relevant technologies. This can be a barrier when implementing JITAIs in older adults, and resource-intense measures might need to be taken to familiarize older adults with the technologies.

\section{Acknowledgements}

We would like to thank Adele Krusche (University of Southampton, UK) for proofreading the final draft of the paper.

Funding: This work was supported by a GetAMoveOn think piece grant from the Engineering and Physical Sciences Research Council (EPSRC), United Kingdom (EP/ N027299/1). 


\section{Footnote}

Conflicts of Interest: The authors have no conflicts of interest to declare.

\section{References}

1. Bloom DE, Canning D, Lubet A. Global population aging: facts, challenges, solutions \& perspectives. Daedalus 2015;144:80-92.

2. United Nations, Department of Economic and Social Affairs, Population Division. World Population Ageing 2013. New York: United Nations Publications, 2013.

3. Christensen K, Doblhammer G, Rau R, et al. Ageing populations: the challenges ahead. Lancet 2009;374:1196-208.

4. Rowe JW. Successful aging of societies. Dædalus 2015;144:5-12.

5. Sargent-Cox KA, Butterworth P, Anstey KJ. Role of physical activity in the relationship between mastery and functional health. Gerontologist 2015;55:120-31.

6. Ziegelmann JP, Knoll N. Future directions in the study of health behavior among older adults. Gerontology 2015;61:469-76.

7. Vogel T, Brechat PH, Leprêtre PM, et al. Health benefits of physical activity in older patients: a review. Int J Clin Pract 2009;63:303-20.

8. Colcombe SJ, Erickson KI, Scalf PE, et al. Aerobic exercise training increases brain volume in aging humans. J Gerontol A Biol Sci Med Sci 2006;61:1166-70.

9. Erickson KI, Gildengers AG, Butters MA. Physical activity and brain plasticity in late adulthood. Dialogues Clin Neurosci 2013;15:99-108.

10. Olanrewaju O, Kelly S, Cowan A, et al. Physical activity in community dwelling older people: a systematic review of reviews of interventions and context. PLoS One 2016;11:e0168614.

11. Elavsky S, McAuley E, Motl RW, et al. Physical activity enhances long-term quality of life in older adults: efficacy, esteem, and affective influences. Ann Behav Med 2005;30:138-45.

12. Sun F, Norman IJ, While AE. Physical activity in older people: a systematic review. BMC Public Health 2013;13:449.

13. Jefferis BJ, Sartini C, Lee IM, et al. Adherence to physical activity guidelines in older adults, using objectively measured physical activity in a population-based study. BMC Public Health 2014;14:382.

14. Harvey JA, Chastin SF, Skelton DA. How sedentary are older people? A systematic review of the amount of sedentary behavior. J Aging Phys Act 2015;23:471-87.

15. Matthews CE, Chen KY, Freedson PS, et al. Amount of time spent in sedentary behaviors in the United States, 2003-2004. Am J Epidemiol 2008;167:875-81.

16. Muellmann S, Forberger S, Möllers T, et al. Effectiveness of eHealth interventions for the promotion of physical activity in older adults: a systematic review protocol. Syst Rev 2016;5:47.

17. Müller AM, Khoo S. Non-face-to-face physical activity interventions in older adults: a systematic review. Int J Behav Nutr Phys Act 2014;11:35.

18. Vandelanotte C, Müller AM, Short CE, et al. Past, present, and future of ehealth and mhealth research to improve physical activity and dietary behaviors. J Nutr Educ Behav 2016;48:219-228.e1.

19. Heart T, Kalderon E. Older adults: are they ready to adopt health-related ICT? Int J Med Inform 2013;82:e209-31.

20. O'Brien T, Troutman-Jordan M, Hathaway D, et al. Acceptability of wristband activity trackers among community dwelling older adults. Geriatr Nurs 2015;36:S21-5.

21. Zhou J, Patrick Rau PL, Salvendy G. Age-related difference in the use of mobile phones. Univ Access Inf Soc 2014;13:401-13.

22. Gell NM, Rosenberg DE, Demiris G, et al. Patterns of technology use among older adults with and without disabilities. Gerontologist 2015;55:412-21.

23. Kurniawan S. Older people and mobile phones: a multi-method investigation. Int J Hum Comput Stud 2008;66:889-901.

24. Parker SJ, Jessel S, Richardson JE, et al. Older adults are mobile too! Identifying the barriers and facilitators to older adults' use of mHealth for pain management. BMC Geriatr 2013;13:43.

25. Ofcom. Adults' media use and attitudes: Ofcom; 2015 [cited 2017 Mar 30]. Available online: https://www.ofcom. org.uk/_data/assets/pdf_file/0014/82112/2015_adults_ media_use_and_attitudes_report.pdf

26. Nahum-Shani I, Smith SN, Tewari A, et al. Just-in-Time Adaptive Interventions (JITAIs): an organizing framework for ongoing health behavior support: (Technical Report No. 14-126). University Park, PA: The Methodology Center, Penn State, 2014.

27. Kim BH, Glanz K. Text messaging to motivate walking in older African Americans: A randomized controlled trial. Am J Prev Med 2013;44:71-5.

28. Müller AM, Khoo S, Morris T. Text messaging for exercise 
promotion in older adults from an upper-middle-income country: Randomized controlled trial. J Med Internet Res 2016;18:e5.

29. Ammann R, Vandelanotte C, de Vries H, et al. Can a website-delivered computer-tailored physical activity intervention be acceptable, usable, and effective for older people? Health Educ Behav 2013;40:160-70.

30. Irvine AB, Gelatt VA, Seeley JR, et al. Web-based intervention to promote physical activity by sedentary older adults: randomized controlled trial. J Med Internet Res 2013;15:e19.

31. Peels DA, van Stralen MM, Bolman C, et al. The differentiated effectiveness of a printed versus a Web-based tailored physical activity intervention among adults aged over 50. Health Educ Res 2014;29:870-82.

32. van Dyck D, Plaete J, Cardon G, et al. Effectiveness of the self-regulation eHealth intervention 'MyPlan1.0.' on physical activity levels of recently retired Belgian adults: a randomized controlled trial. Health Educ Res 2016;31:653-64.

33. Wijsman CA, Westendorp RG, Verhagen EA, et al. Effects of a web-based intervention on physical activity and metabolism in older adults: Randomized controlled trial. J Med Internet Res 2013;15:e233.

34. Lara J, O'Brien N, Godfrey A, et al. Pilot randomised controlled trial of a web-based intervention to promote healthy eating, physical activity and meaningful social connections compared with usual care control in people of retirement age recruited from workplaces. PLoS One 2016;11:e0159703.

35. Mouton A, Cloes M. Efficacy of a web-based, center-based or combined physical activity intervention among older adults. Health Educ Res 2015;30:422-35.

36. Silveira $\mathrm{P}$, van de Langenberg $\mathrm{R}$, van Het Reve E, et al. Tablet-based strength-balance training to motivate and improve adherence to exercise in independently living older people: a phase II preclinical exploratory trial. J Med Internet Res 2013;15:e159.

37. Cadmus-Bertram LA, Marcus BH, Patterson RE, et al. Randomized trial of a fitbit-based physical activity intervention for women. Am J Prev Med 2015;49:414-8.

38. Thompson WG, Kuhle CL, Koepp GA, et al. "Go4Life" exercise counseling, accelerometer feedback, and activity levels in older people. Arch Gerontol Geriatr 2014;58:314-9.

39. Peels DA, Bolman C, Golsteijn RH, et al. Long-term efficacy of a printed or a Web-based tailored physical activity intervention among older adults. Int J Behav Nutr Phys Act 2013;10:104.

40. Hekler EB, Michie S, Pavel M, et al. Advancing models and theories for digital behavior change interventions. Am J Prev Med 2016;51:825-32.

41. Spruijt-Metz D, Hekler E, Saranummi N, et al. Building new computational models to support health behavior change and maintenance: new opportunities in behavioral research. Transl Behav Med 2015;5:335-46.

42. Patrick K, Hekler EB, Estrin D, et al. The pace of technologic change: Implications for digital health behavior intervention research. Am J Prev Med 2016;51:816-24.

43. Nahum-Shani I, Smith SN, Spring BJ, et al. Just-in-Time Adaptive Interventions (JITAIs) in mobile health: Key components and design principles for ongoing health behavior support. Ann Behav Med 2016. [Epub ahead of print].

44. Nahum-Shani I, Hekler EB, Spruijt-Metz D. Building health behavior models to guide the development of justin-time adaptive interventions: a pragmatic framework. Health Psychol 2015;34S:1209-19.

45. Bond DS, Thomas JG, Raynor HA, et al. B-MOBILE-a smartphone-based intervention to reduce sedentary time in overweight/obese individuals: a within-subjects experimental trial. PLoS One 2014;9:e100821.

46. King AC, Hekler EB, Grieco LA, et al. Harnessing different motivational frames via mobile phones to promote daily physical activity and reduce sedentary behavior in aging adults. PLoS One 2013;8:e62613.

47. King AC, Hekler EB, Grieco LA, et al. Effects of three motivationally targeted mobile device applications on initial physical activity and sedentary behavior change in midlife and older adults: A randomized trial. PLoS One 2016;11:e0156370.

48. Hekler EB, Klasnja P, Traver V, et al. Realizing effective behavioral management of health: the metamorphosis of behavioral science methods. IEEE Pulse 2013;4:29-34.

49. World Health Organization. Global recommendations on physical activity for health. Geneva, Switzerland; 2010.

50. Sparling PB, Howard BJ, Dunstan DW, et al. Recommendations for physical activity in older adults. BMJ 2015;350:h100.

51. Greenwood-Hickman MA, Renz A, Rosenberg DE. Motivators and barriers to reducing sedentary behavior among overweight and obese older adults. Gerontologist 2016;56:660-8.

52. Heckhausen J, Wrosch C, Schulz R. A motivational theory of life-span development. Psychol Rev 2010;117:32-60.

53. García-Esquinas E, Andrade E, Martínez-Gómez D, et al. Television viewing time as a risk factor for frailty and functional limitations in older adults: results from 2 
European prospective cohorts. Int J Behav Nutr Phys Act 2017;14:54.

54. de Rezende LF, Rey-López JP, Matsudo VKR, et al. Sedentary behavior and health outcomes among older adults: a systematic review. BMC Public Health 2014;14:333.

55. Healy GN, Dunstan DW, Salmon J, et al. Breaks in sedentary time: beneficial associations with metabolic risk. Diabetes Care 2008;31:661-6.

56. Owen N, Healy GN, Matthews CE, et al. Too much sitting: the population health science of sedentary behavior. Exerc Sport Sci Rev 2010;38:105-13.

57. Maher JP, Sliwinski MJ, Conroy DE. Feasibility and preliminary efficacy of an intervention to reduce older adults' sedentary behavior. Transl Behav Med 2017;7:52-61.

58. Rutten GM, Savelberg HH, Biddle SJ, et al. Interrupting long periods of sitting: good STUFF. Int J Behav Nutr Phys Act 2013;10:1.

59. Gardner B, Smith L, Lorencatto F, et al. How to reduce sitting time? A review of behaviour change strategies used in sedentary behaviour reduction interventions among adults. Health Psychol Rev 2016;10:89-112.

60. Devereux-Fitzgerald A, Powell R, Dewhurst A, et al. The acceptability of physical activity interventions to older adults: A systematic review and meta-synthesis. Soc Sci Med 2016;158:14-23.

61. Gellert P, Ziegelmann JP, Warner LM, et al. Physical activity intervention in older adults: Does a participating partner make a difference? Eur J Ageing 2011;8:211-9.

62. McAuley E, Jerome GJ, Elavsky S, et al. Predicting longterm maintenance of physical activity in older adults. Prev Med 2003;37:110-8.

63. Lindsay Smith G, Banting L, Eime R, et al. The association between social support and physical activity in older adults: a systematic review. Int J Behav Nutr Phys Act 2017;14:56.

64. van Stralen, MM, De Vries H, Mudde AN, et al. Determinants of initiation and maintenance of physical activity among older adults: a literature review. Health Psychol Rev 2009;3:147-207.

65. Hadgraft NT, Winkler EA, Healy GN, et al. Intervening to reduce workplace sitting: mediating role of socialcognitive constructs during a cluster randomised controlled trial. Int J Behav Nutr Phys Act 2017;14:27.

66. van Dantzig S, Geleijnse G, van Halteren AT. Toward a persuasive mobile application to reduce sedentary behavior. Pers Ubiquit Comput 2013;17:1237-46.

67. Henson J, Davies MJ, Bodicoat DH, et al. Breaking up prolonged sitting with standing or walking attenuates the postprandial metabolic response in postmenopausal women: a randomized acute study. Diabetes Care 2016;39:130-8.

68. Lally P, van Jaarsveld CH, Potts HW, et al. How are habits formed: Modelling habit formation in the real world. Eur J Soc Psychol 2010;40:998-1009.

69. Fitzsimons CF, Kirk A, Baker G, et al. Using an individualised consultation and activPAL ${ }^{\mathrm{TM}}$ feedback to reduce sedentary time in older Scottish adults: results of a feasibility and pilot study. Prev Med 2013;57:718-20.

70. Gardiner PA, Clark BK, Healy GN, et al. Measuring older adults' sedentary time: reliability, validity, and responsiveness. Med Sci Sports Exerc 2011;43:2127-33.

71. Gennuso KP, Thraen-Borowski KM, Gangnon RE, et al. Patterns of sedentary behavior and physical function in older adults. Aging Clin Exp Res 2016;28:943-50.

72. Gardiner PA, Eakin EG, Healy GN, et al. Feasibility of reducing older adults' sedentary time. Am J Prev Med 2011;41:174-7.

73. Gardner B, Lally P, Wardle J. Making health habitual: the psychology of 'habit-formation' and general practice. Br J Gen Pract 2012;62:664-6.

74. Rosenberg DE, Gell NM, Jones SM, et al. The feasibility of reducing sitting time in overweight and obese older adults. Health Educ Behav 2015;42:669-76.

75. PEW Research Centre. Mobile Fact Sheet: PEW Research Centre; 2017 [cited 2017 Mar 30]. Available online: URL: http://www.pewinternet.org/fact-sheet/mobile/

76. Statistica. UK: smartphone ownership by age from 20122016: Statistica; 2016 [cited 2017 Mar 30]. Available online: https://www.statista.com/statistics/271851/ smartphone-owners-in-the-united-kingdom-uk-by-age/

77. Lyons EJ, Swartz MC, Lewis ZH, et al Feasibility and acceptability of a wearable technology physical activity intervention with telephone counseling for mid-aged and older adults: a randomized controlled pilot trial. JMIR Mhealth Uhealth 2017;5:e28.

78. McMahon SK, Lewis B, Oakes M, et al. Older adults' experiences using a commercially available monitor to self-track their physical activity. JMIR Mhealth Uhealth 2016;4:e35.

79. Carstensen LL, Isaacowitz DM, Charles ST. Taking time seriously: A theory of socioemotional selectivity. Am Psychol 1999;54:165-81.

80. Charles ST, Carstensen LL. Social and emotional aging. Annu Rev Psychol 2010; 61:383-409.

81. Consolvo S, McDonald DW, Toscos T, et al. Activity 
sensing in the wild: a field trial of ubifit garden. In: The 26th Annual CHI Conference on Human Factors in Computing Systems, CHI 2008: Conference proceedings; April 5-10, 2008 in Florence, Italy. NY: ACM, 2008;1797-1806.

82. Barone Gibbs B, Brach JS, Byard T, et al. Reducing sedentary behavior versus increasing moderate-to-vigorous intensity physical activity in older adults. J Aging Health 2016;29:247-67.

doi: 10.21037/mhealth.2017.08.05

Cite this article as: Müller AM, Blandford A, Yardley L. The conceptualization of a just-in-time adaptive intervention (JITAI) for the reduction of sedentary behavior in older adults. mHealth 2017;3:37.
83. Reinwand DA, Schulz DN, Crutzen R, et al. Who follows ehealth interventions as recommended? A study of participants' personal characteristics from the experimental arm of a randomized controlled trial. J Med Internet Res 2015;17:e115.

84. Spruijt-Metz D, Nilsen W. Dynamic models of behavior for Just-in-Time Adaptive Interventions. IEEE Pervasive Comput 2014;13:13-7. 


\section{Supplementary}

Table S1 JITAI for reducing sitting time in older adults

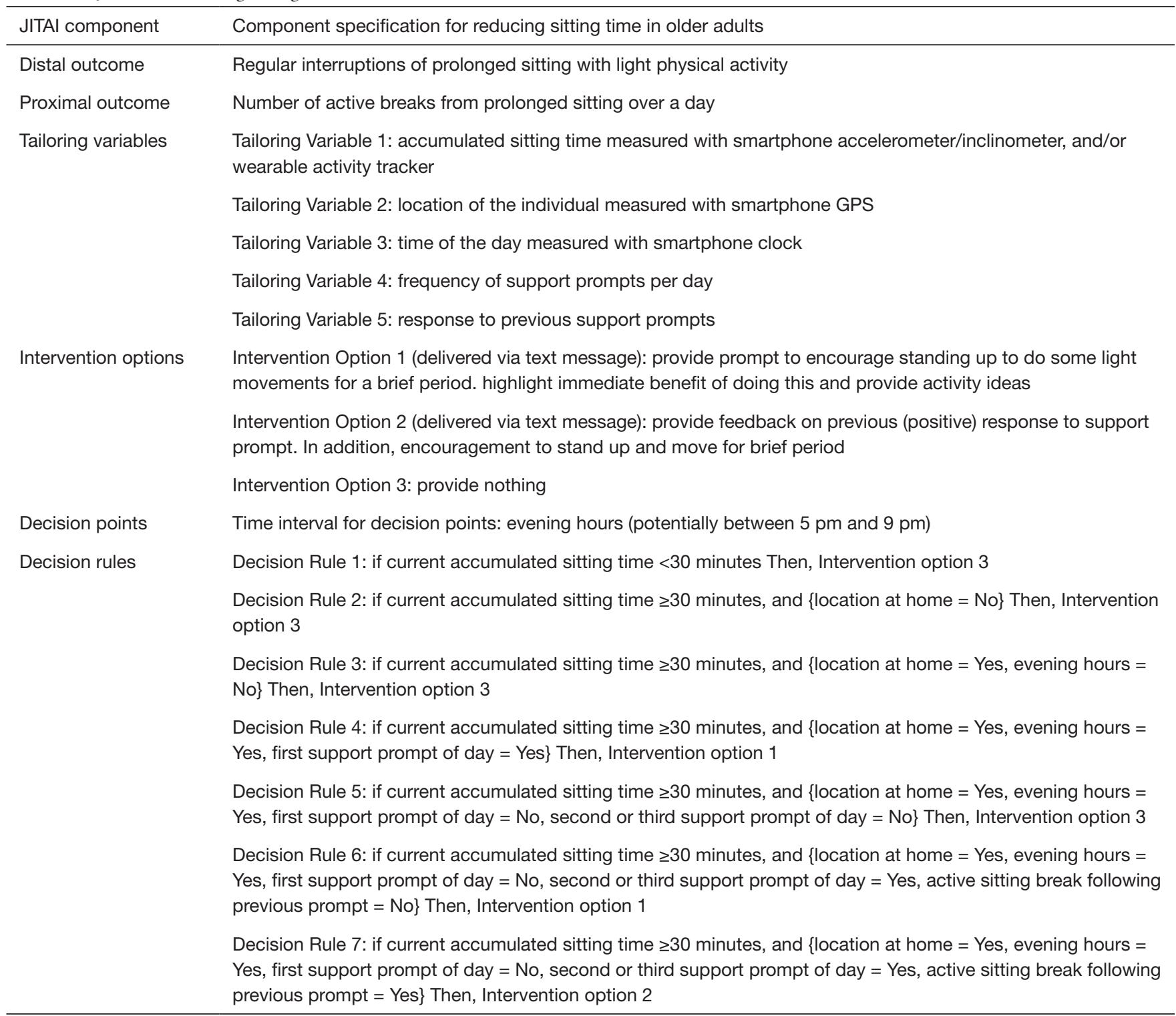

JITAI, Just-In-Time Adaptive Intervention. 